\title{
LETTERS
}

\section{Healthcare Experiences of Autistic Adults}

\author{
Bryan K. Woodruff, MD
}

Mayo Clinic Arizona, Scottsdale, AZ, USA

$\mathrm{J}$ Gen Intern Med 28(7):870

DOI: $10.1007 / \mathrm{s} 11606-013-2428-y$

(c) Society of General Internal Medicine 2013

To the Editors: I would like to applaud Dr. Nicolaidis and colleagues for addressing an understudied topic in an understudied population - healthcare disparities experienced by autistic adults. ${ }^{1}$ The inclusion of autistic individuals as equal members of the study team is an ideal example of community-based participatory research. Such efforts answer calls for better understanding of issues impacting autistic adults. ${ }^{2}$ The key finding that despite similar access to a primary care provider, autistic adults are less likely than their non-autistic peers to receive important preventive healthcare measures is unfortunate, but not surprising. Incorporation of variables such as race/ethnicity, income and type of health insurance that could potentially confound the study findings further strengthens this study.

Several aspects of the study merit clarification. First, it is unclear why the authors oversampled adults with disabilities in their non-autistic sample if their intent was to identify differences between autistics and non-autistics. The nonautistic sample, once enriched by individuals with disabilities, by definition might be skewed towards responses shared by disabled populations in general.

Second, it would be helpful to know how the study was advertised on the Gateway Project to potential participants. For example, if the study was labeled as an "Autism Study", it might attract participants with potentially biased responses, such as family members of autistic individuals. This could be particularly relevant when one considers that such relatives may exhibit subclinical autistic traits themselves. $^{3}$
It is interesting that only $71 \%$ of the autistic cohort identified as having a disability. The large percentage of participants diagnosed with Asperger's disorder is also unusual when that diagnosis represents a minority of the autism spectrum disorders based on prior prevalence estimates. ${ }^{4}$ The authors point out, but do not provide a compelling explanation for, the excess of female participants in the study, other than that female participants tend to be overrepresented in online surveys. This is particularly striking when one considers the 4:1 male predominance reported for autism spectrum disorders. ${ }^{5}$

The critiques outlined above are minor, and this survey hopefully is the first of many to identify the gaps in healthcare services for a subset of the adult population that has for years gone largely unrecognized. It behooves us as a society to recognize and address those disparities to optimize the health of autistic individuals across their lifespan.

Corresponding Author: Bryan K. Woodruff, MD; Mayo Clinic Arizona, 13400 East Shea Blvd., Scottsdale, AZ 85259, USA (e-mail: Woodruff.bryan@mayo.edu).

\section{REFERENCES}

1. Nicolaidis C, et al. Comparison of healthcare experiences in autistic and non-autistic adults: a cross-sectional online survey facilitated by an Academic-Community Partnership. J Gen Intern Med. 2012. doi: $10.1007 /$ s11606-012-2262-7.

2. Piven J, Rabins P, G. Autism-in-Older Adults Working. Autism spectrum disorders in older adults: toward defining a research agenda. J Am Geriatr Soc. 2011;59(11):2151-5.

3. Sucksmith E, Roth I, Hoekstra RA. Autistic traits below the clinical threshold: re-examining the broader autism phenotype in the 21 st century. Neuropsychol Rev. 2011;21(4):360-89.

4. Fombonne E. Epidemiology of autistic disorder and other pervasive developmental disorders. J Clin Psychiatry. 2005;66(Suppl 10):3-8.

5. Giarelli E, et al. Sex differences in the evaluation and diagnosis of autism spectrum disorders among children. Disabil Health J. 2010;3(2):107-16. 